\title{
Origin of Ubiquitous Stripes at the Graphite-Water Interface
}

\author{
Sebastian Seibert, Stefanie Klassen, Annamaria Latus, Ralf Bechstein, and Angelika Kühnle*
}

Cite This: Langmuir 2020, 36, 7789-7794

Read Online

ABSTRACT: The investigation of solid-liquid interfaces is pivotal for understanding processes like wetting, corrosion, and mineral dissolution and growth. The graphite-water interface constitutes a prime example for studying the water structure at a seemingly hydrophobic surface. Surprisingly, in a large number of atomic force microscopy (AFM) experiments, well-ordered stripes have been observed at the graphite-water interface. Although many groups have reported on the observation of stripes at this interface, fundamental properties and, in particular, the origin of the stripes are still under debate. Proposed origins include contamination, interplanar stacking of graphene layers, formation
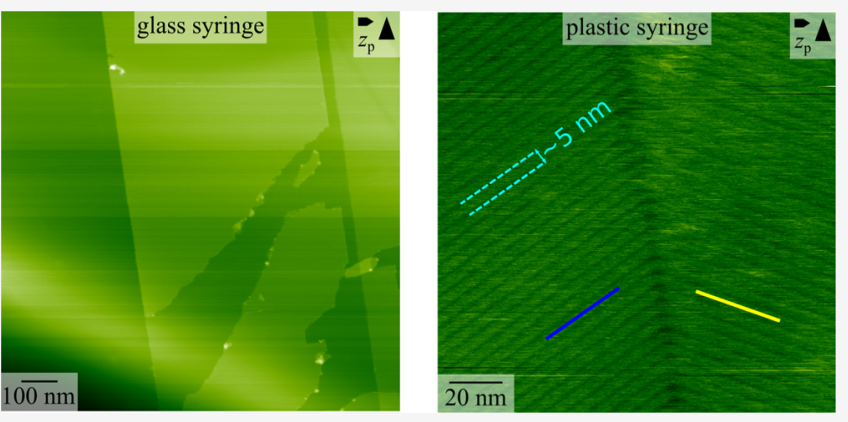
of methanol-water nanostructures, and adsorption of nitrogen molecules. Especially, the latter interpretation has received considerable attention because of its potential impact on explaining the long-range nature of the hydrophobic interaction. In this study, we demonstrate that these stripes readily form when using standard plastic syringes to insert the water into the AFM instrument. In contrast, when clean glass syringes are used instead, no such stripes form even though nitrogen was present. We, therefore, conclude that contaminations from the plastic syringe rather than nitrogen constitute the origin of the stripes we observe. We provide high-resolution AFM data that reveal detailed structural insights into the arrangement of the stripes. The rich variability of our data suggests that the stripes might be composed of several different chemical species. Still, we cannot rule out that the stripes observed in the literature might originate from other sources; our study offers a rather straightforward explanation for the origin of the stripes. In the view of these results, we propose to carefully reconsider former assignments.

\section{INTRODUCTION}

The graphite-water interface constitutes a prime example for studying the water structure at a seemingly simple model surface. Besides the fundamental interest in elucidating the water structure at a surface that is generally believed to be hydrophobic, ${ }^{1}$ the graphite/graphene surface also serves as a model system for many application-related studies. ${ }^{2-4}$ In the past, an ever-growing number of research groups have reported atomic force microscopy (AFM) experiments on the formation of well-ordered stripes on the graphite/graphene surface. ${ }^{5-18}$ Despite their ubiquitous appearance, the origin and many structural details of these stripes are still under debate. For example, the stripes have been reported to order in domains with different orientations with respect to each other. ${ }^{5-16}$ Moreover, different stripe widths ranging from 2 to $6 \mathrm{~nm}$ have been reported in literature. ${ }^{5-7,12,15}$ Similarly, contradicting information is available on the solvation structure on top of the stripes. ${ }^{17,19}$ Although some groups have ascribed the stripes to airborne contaminations ${ }^{11-14}$ or to the formation of methanol-water nanostructures, ${ }^{18}$ others have explained the origin of the stripes by intercalation and different interplanar stackings $^{12}$ or the assembly of dissolved nitrogen molecules at the graphite/graphene-water interface. ${ }^{5-9,17}$ The interpretation of the stripes being composed of nitrogen has received considerable attention because of its potential impact for explaining the long-range nature of the hydrophobic interaction. $^{17,20,21}$ In the view of this discussion, it becomes increasingly relevant to shed light onto the structural details of the stripes and, in particular, to clarify the chemical nature of the constituents.

Here, we present an AFM study of stripe-like adsorbates at the graphite-water interface (in the following referred to as stripes). We demonstrate that these stripes are present when standard plastic syringes are used to place the water droplet into the instrument. When avoiding plastic syringes and using clean glass syringes instead, we do not observe the stripes even though nitrogen was present. We, therefore, conclude that the stripes observed in our experiments are composed of species that originate from the plastic syringes. Our observations suggest that at least some of the stripes reported in the literature could have the same straightforward origin. There-

Received: March 17, 2020

Revised: June 22, 2020

Published: June 23, 2020 
fore, we provide a detailed structural investigation of the stripes as a reference for comparison with existing and future work exhibiting stripes at the graphite-water interface. The structural features reported here, including the domain orientation, stripe widths, internal structure and solvation structure, exhibit rich variability. This variability, which is in excellent agreement with structural investigations of the stripes reported in the literature, suggests that the contamination is composed of different chemical species. Although we cannot exclude that the stripes from previous reports might have yet another origin, we recommend to reconsider earlier assignments to nitrogen in the view of our results.

\section{EXPERIMENTAL SECTION}

All data shown in this study were measured on highly oriented pyrolytic graphite purchased from PLANO GmbH (Germany). Prior to most measurements, the graphite surface was freshly cleaved with an adhesive tape. In few cases, the graphite was kept in air for some time before exposing it to aqueous solution. The images obtained after the latter procedure look the same as those for the freshly cleaved surface. The ultrapure water used in this study was obtained from two different purification setups (Merck Millipore, Germany, and Stakpure GmbH, Germany). As it is sometimes suggested that AFM imaging conditions improve in the presence of ions, we also performed some experiments using sodium chloride solutions. The used aqueous solutions were produced by dissolving sodium chloride (Carl Roth $\mathrm{GmbH}+$ Co. KG, Germany) in ultrapure water. The presence of the ions does not change the observations made here. To insert the solutions into the instrument, two different kinds of syringes were used. For the "standard" measurement, we use plastic syringes (Braun Melsungen AG, Germany). As a reference, we used syringes made of glass (Poulten \& Graf GmbH, Germany). In some experiments, we intended to clean the glass syringes with a surfactant solution (Hellmanex III, Sigma-Alrdich, Germany). The surfactant solution is composed of phosphates, anionic surfactants, and nonionic surfactants.

All AFM measurements shown here were performed in the frequency-modulation mode. Two different AFM instruments were used. The first setup used is based on a Multimode AFM instrument with a Nanoscope V controller (Bruker, Nano Surfaces Division, USA) and was modified to improve the signal-to-noise ratio ${ }^{22}$ and to enable three-dimensional (3D) mapping ${ }^{23}$ with photothermal excitation. ${ }^{24}$ The second setup used is a Cypher ES AFM instrument from Asylum Research, an Oxford Instruments Company, USA. Silicon cantilevers (TAP300 GD-G, Budget Sensors, Bulgaria) with a gold coating on the backside were used. According to the manufacturer, the resonance frequency is $300 \mathrm{kHz}$ (typical range: $200-400 \mathrm{kHz}$ ) in air and the force constant is $40 \mathrm{~N} / \mathrm{m}$ (typical range: $20-75 \mathrm{~N} / \mathrm{m}$ ). The eigenfrequency of the cantilever in water is approximately $100-150 \mathrm{kHz}$. For 3D AFM experiments, the amplitude of oscillation was $0.05-0.1 \mathrm{~nm}$. For two-dimensional (2D) AFM experiments, the amplitude was $0.05-1 \mathrm{~nm}$. In the upper right corner of all $2 \mathrm{D}$ AFM images shown in this study, the recorded channel $\left(z_{\mathrm{p}}: z\right.$-piezo displacement, $\Delta \nu$ : frequency shift) and the slow and fast scan directions are displayed. The color scale ranges from dark (low value) to bright (high value).

\section{RESULTS AND DISCUSSION}

For investigating the graphite-water interface with AFM, a graphite sample is cleaved and inserted into the liquid cell of the instrument. The solution is then placed on top of the graphite surface using a syringe. In a "standard" experiment, we use standard aseptic syringes for clinical use, which are made of polypropylene (barrel) and polyethylene (plunger). When performing AFM imaging after the solution was inserted with such a plastic syringe, well-ordered stripes are observed in most of the cases. Altogether, we performed more than 100 independent standard experiments and approximately, 80\% of them showed stripes. During the other $20 \%$ of the experiments, no stripe formation was observed. Figure 1a
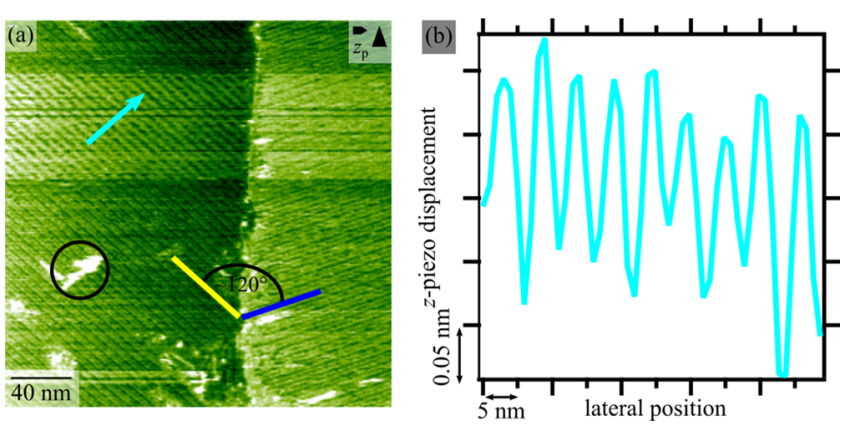

Figure 1. (a) Drift-corrected AFM image of a stripe-covered graphite surface taken in water. (b) Height profile taken from (a) (position is indicated by the cyan arrow), revealing a stripe width of approximately $5 \mathrm{~nm}$. The displayed height profile is an average over ten height profiles.

shows a representative AFM image taken at the graphitewater interface with the surface being entirely covered by the stripes. Two different stripe domains are visible, which are separated by a step edge. At some positions, bright protrusion can be seen on top of the stripes (black circle in Figure 1a), the nature of which is unclear to us. The height profile extracted from Figure 1a (position is indicated by the cyan arrow) shown in Figure 1b, reveals a stripe width of approximately $5 \mathrm{~nm}$.

We note that contaminations from disposable needles have been reported earlier to be the source of adsorbates at the graphite-water interface. $^{25}$ In this latter work, however, nanobubble-like objects have been observed, which have been ascribed to polydimethylsiloxane.

In sharp contrast, when we avoided plastic syringes, the stripes were absent. More specifically, when inserting the water with a clean glass syringe, we never observed stripes. As we do not observe stripes in about $1 / 5$ of all experiments using plastic syringes, the experiment with glass syringes was repeated seven times to provide solid experimental evidence that no stripes are seen when glass syringes are used. In these experiments, nitrogen was present; therefore, we cannot confirm the results presented in the literature, which assign the stripes to nitrogen. Interestingly, we also performed experiments in which we first injected water with a glass syringe and afterward exchanged the water using a plastic syringe. After injecting the water with the glass syringe, a stripe-free interface was observed (Figure 2a). In this image, the graphite surface with step edges can be seen. Interestingly, after exchanging the water via a plastic syringe, stripes start to form on the surface almost immediately. In Figure $2 b$, the same surface area is seen as that in Figure 2a; however, now stripes cover the terraces. A zoom onto the terrace and the step edge in the upper right corner is given in Figure $2 c$ to clearly present the stripes. A height profile taken at the indicated position in Figure $2 \mathrm{c}$ is given in Figure $2 \mathrm{~d}$, revealing stripes with a width of approximately $5 \mathrm{~nm}$. The latter experiment (first injecting the water with a glass syringe and then replacing it using a plastic syringe) was performed two times with identical results. In another experiment, we first "cleaned" the glass syringes with a surfactant solution. Upon inserting the water droplet with such a precleaned glass syringe, stripes are again formed at the graphite-water interface. This finding strongly suggests that in this case, 

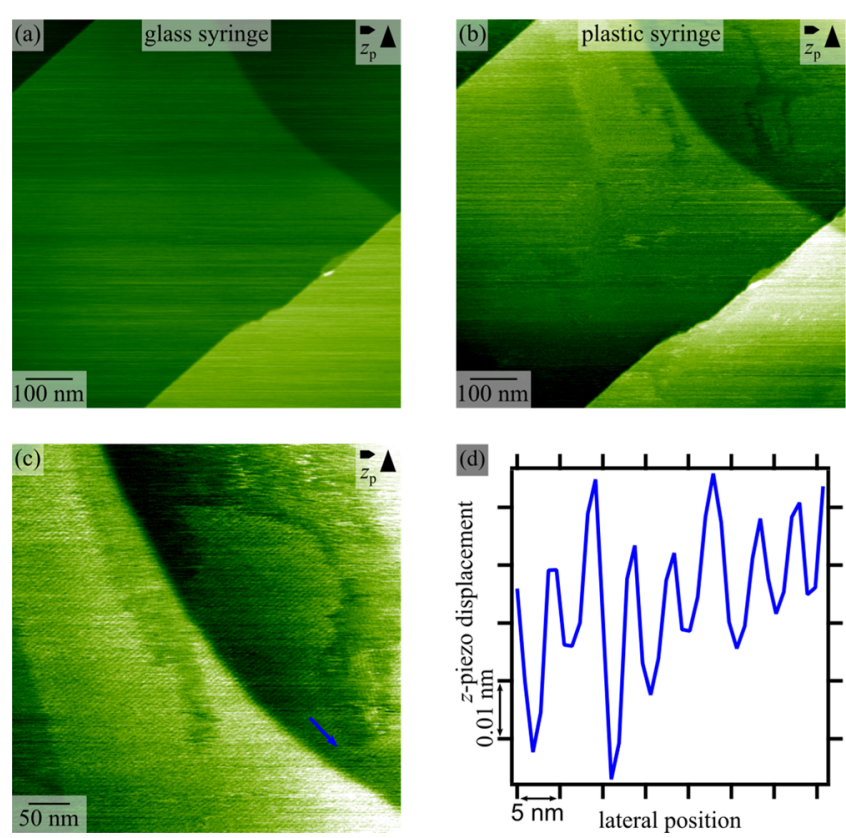

Figure 2. AFM images taken at the graphite-water interface. (a) Glass syringe was used to inject pure water directly onto the sample. The bare graphite surface is observed, exhibiting terraces and step edges. (b) When exchanging the water in situ using a plastic syringe, adsorbates become visible at the graphite-water interface. (c) Zoom image of (b) reveals stripes at the interface. (d) Displayed height profile is extracted from (c) (position is marked by a blue arrow). The stripe width is approximately $5 \mathrm{~nm}$. The height profile is an average over 15 height profiles.

stripes form from the organic surfactant molecules that seem to remain in the syringe even after rinsing it with water.

In the following section, we will discuss structural properties of the stripes observed in our experiments as a reference to existing and future work presenting stripes at the graphitewater interface. Our analysis includes the domain orientation, the stripe widths, and the internal as well as the solvation structure of the stripes.

Domain Orientations. In agreement with what is reported in the literature, we observe domains of stripes with three different orientations that exhibit the well-known $120^{\circ}$ rotation. $5,6,9,10,12,13,15$ This is expected due to the threefold rotational symmetry of the graphite surface. In Figure 1a, two of the three domain orientations can be seen, marked by a yellow and blue line. However, we also observe domains that exhibit orientations different from $120^{\circ}$ with respect to each other. Figure 3 displays two other domains, which are rotated only by approximately $6^{\circ}$ with respect to each other. The observed angle also differs from $30^{\circ}$, which could be expected from the zigzag and arm-chair direction on the graphite surface. ${ }^{7}$ Because of the occurrence of different stripe orientations in a single image, a scan artefact can be ruled out.

A possible explanation for the observed $6^{\circ}$ angle might be the rotation of the top graphene layer, forming a moire pattern because of cleaving of the graphite surface. ${ }^{11,26,27}$ Defects such as dislocations or grain boundaries in the graphite lattice can also lead to misorientation and, therefore, maybe to different domain orientations. ${ }^{11,28}$ However, in many other images, we also observe rotation angles different from 120 or $30^{\circ}$ (e.g., approximately $132,107,55,157$, and $13^{\circ}$; see the Supporting Information Figure S1). This finding is an indication of the fact

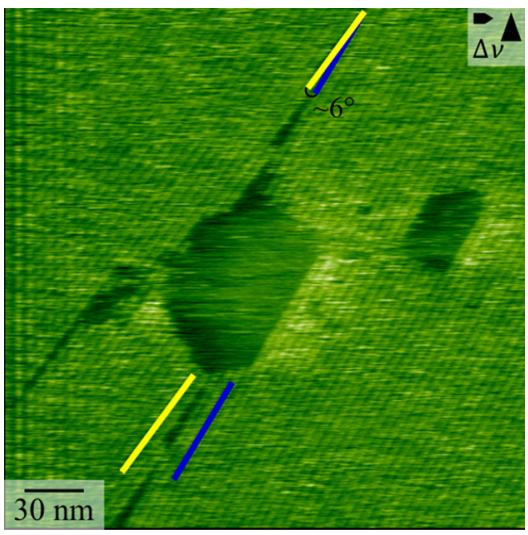

Figure 3. Drift-corrected AFM image of a stripe-covered graphite surface taken in water. Two domains of stripes with different orientations with respect to each other are visible. The blue and yellow lines highlight the orientation of the individual domains. The enclosed angle of approximately $6^{\circ}$ deviates from the $120^{\circ}$ rotation expected for rotational domains on graphite. The vertical lines to the left of the image are caused by the distance feedback loop due to the high scan speed.

that the domain orientations do not depend on the graphite surface as a template solely. Furthermore, the domains can change their orientation on a time scale of minutes (see the Supporting Information Figure S2). This result demonstrates that the observed domain orientations are not simply given by the threefold rotational symmetry of the graphite surface.

Stripe Widths. In the literature, stripes with widths ranging from 2 to $6 \mathrm{~nm}$ are reported..$^{5-7,12,15}$ In Figure 4a, a further
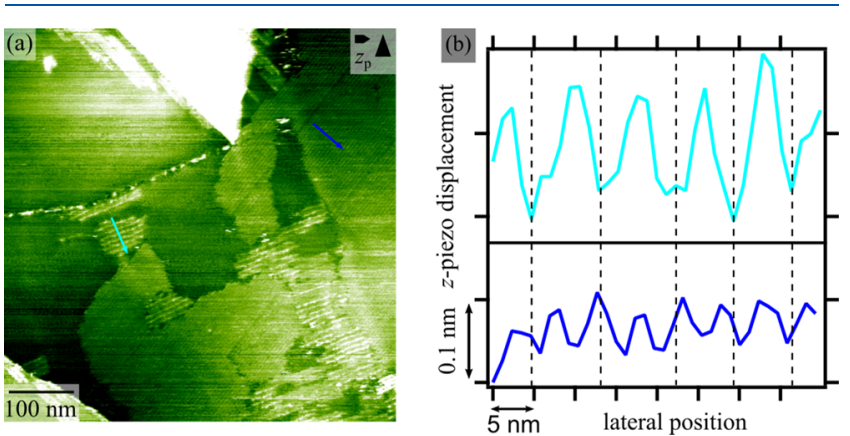

Figure 4. (a) AFM image of a stripe-covered graphite surface taken in water. Islands of stripes with different stripe widths can be observed at the interface. (b) Displayed height profiles are extracted from (a) at the positions indicated by arrows. The height profiles are averaged over seven height profiles.

image taken at the graphite-water interface is shown. In this image, islands of stripes with different widths coexist at the interface. To discuss the different stripe widths in more detail, height profiles are extracted from two different islands with different stripe widths. The blue profile in Figure $4 \mathrm{~b}$ reveals a stripe width of approximately $5 \mathrm{~nm}$. In contrast to that, the cyan profile reveals a stripe width of approximately $8 \mathrm{~nm}$. Interestingly, images with different stripe widths within a single frame have been reported before in the literature. ${ }^{6,12}$ These different widths might indicate different species being responsible for the stripe formation. We note that different stripe widths might also be associated with multilayer islands of different heights (see the Supporting Information Figure S3). However, as the number of layers seems to be the same for the 
stripe islands shown in Figure 4a, we discard this option for explaining the different stripe widths in the present image.

Inner Structure of the Stripes. In the following, we focus on the inner structure of the stripes. In few instances, we succeeded to obtain high-resolution images that unravel the molecular level structure of the stripes. In these cases, the seemingly homogenous stripes exhibit an inner structure that is more complex than that expected given the well-ordered appearance at low resolution (Figure 5a). As most of the
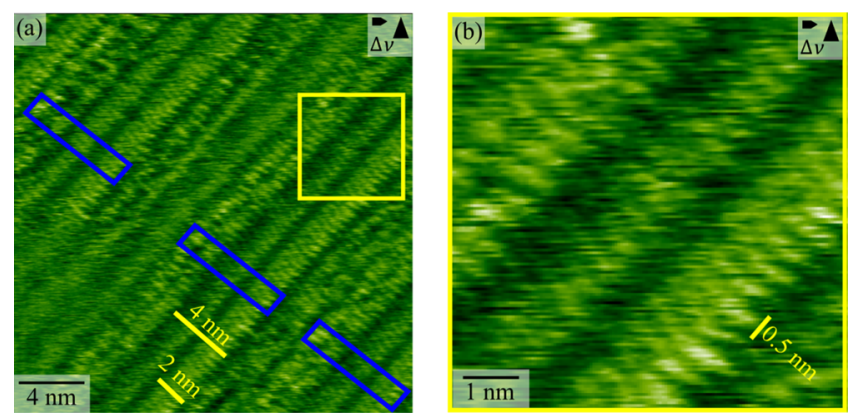

Figure 5. (a) Drift-corrected, high-resolution AFM image of the stripes. (b) Zoom-in image taken at the position indicated by the yellow box in (a). The inner structure is composed of lines with a periodicity of approximately $0.5 \mathrm{~nm}$ along the stripe direction.

stripes reported in the literature have a stripe width of $4 \mathrm{~nm}$, yellow bars of 2 and $4 \mathrm{~nm}$ widths are drawn into the image. Interestingly, when assuming $2 \mathrm{~nm}$ wide stripes, neighboring stripes do not seem to be identically constructed. However, recurring units of stripes can be recognized (blue rectangles). To better characterize the inner structure of the stripes, a zoom-in image of Figure 5a (taken in the area marked with a yellow box) is shown in Figure 5b. The inner structure of the stripes is composed of lines that are nearly perpendicular to the stripe direction. The lines show a periodicity of approximately $0.5 \mathrm{~nm}$ along the stripe direction. This periodicity is similar to the inner structure reported in previous studies. ${ }^{17,18,29}$ Even though neighboring stripes seem to be constructed differently, the periodicity within the stripes is approximately the same for all stripes.

Solvation Structure. For understanding surface processes and reactivity, the specific solvation structure is crucial. In this context, it is highly relevant to evaluate the impact of the presence of the stripes on the solvation structure at the graphite-water interface. So far, only two studies have explicitly investigated the solvation structure on top of the stripes. Interestingly, from these two investigations, contradicting information exists as to whether or not laterally and vertically ordered solvation layers form. Schlesinger and Sivan ${ }^{17}$ have observed both vertical and lateral order on top of the stripes. Yang et al., ${ }^{19}$ on the other hand, have not revealed ordering on top of the stripes. In the absence of stripes, the graphite-water interface shows a vertical but no lateral order. ${ }^{30,31}$ Recording such distance-dependent data is challenging. Therefore, it is well-possible that an existing order is not detected in every experiment, explaining the discrepancy in the literature data. To address this challenge, we compare full 3D frequency shift maps at the graphite-water interface in the absence and in the presence of the well-ordered stripes.

Figure 6a displays a vertical slice of the frequency shift data at the graphite-water interface in the absence of stripes. A vertical but no lateral order can be observed. This is in
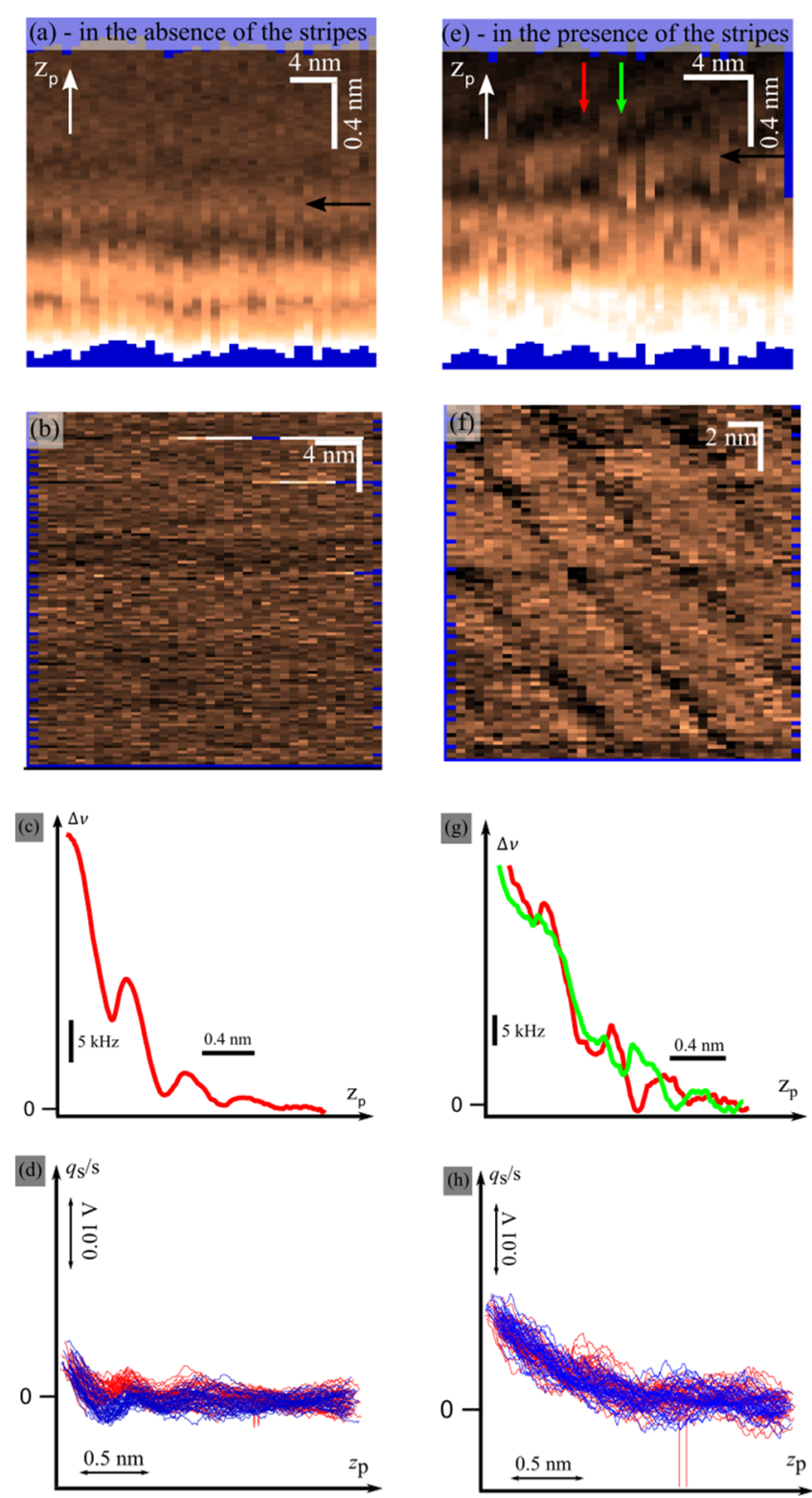

Figure 6. 3D AFM data obtained at the graphite-water interface in the absence $(a-d)$ and in the presence of the stripes $(e-h)$. $(a, e)$ Vertical slices extracted perpendicular to the graphite surface. $(b, f)$ Lateral slices extracted parallel to the graphite surface. The black arrows in (a) and in (e) indicate the positions where the lateral slices are extracted. $(c, g)$ Frequency shift $(\Delta \nu)$ vs $z$-piezo displacement $\left(z_{\mathrm{p}}\right)$ curves. The curve shown in (c) is an average over all experimental 3D data displayed in (a). Site-specific curves in ( $g$ ) are extracted from (e). The red and green arrow in (e) indicates the respective positions. $(\mathrm{d}, \mathrm{h})$ Static deflection raw data as a function of the $z$-piezo displacement.

agreement with previous results. ${ }^{30,31}$ Accordingly, a lateral slice (Figure 6b) through the 3D set reveals no lateral structure in the entire investigated area. The solvation layer-to-layer distance is approximately $0.4 \mathrm{~nm}$ (Figure 6c); thus, a bit larger than the solvation layer-to-layer distance of $0.3 \mathrm{~nm}$ is reported for the calcite-water interface. ${ }^{32}$

In the following part, we will discuss the frequency shift map at the graphite-water interface in the presence of the stripes. Figure $6 \mathrm{e}-\mathrm{h}$ depict the same quantities as Figure $6 \mathrm{a}-\mathrm{d}$. In contrast to the solvation structure in the absence of the stripes, 
a vertical but also a lateral order can be recognized in the presence of the stripes. This vertical and lateral order extends up to $2 \mathrm{~nm}$ in the $z$ direction (Figure 6e). The distance between the lateral maxima is approximately $4 \mathrm{~nm}$, in agreement with the width of the stripes. Figure $6 \mathrm{f}$ displays a lateral slice parallel to the graphite surface (more lateral slices parallel to the graphite surface at different positions are shown in the Supporting Information Figure S4). A clear stripe-like pattern with a width of approximately $4 \mathrm{~nm}$ is visible. The layer-to-layer distance is approximately $0.4 \mathrm{~nm}$ (Figure $6 \mathrm{~g}$ ), similar to the solvation layer-to-layer distance in the absence of the stripes in Figure $6 \mathrm{c}$. The red and green curves are extracted at two different positions in Figure 6e as indicated by the red and green arrow. The maxima of the green curve are a bit shifted compared to the maxima of the red curve.

Two possible ways to interpret the data in the presence of the stripes exist. First, the data given in Figure $6 \mathrm{e}-\mathrm{h}$ show the hydration structure on top of the stripes. Second, the data show the stacked layers of the stripes. The fact that the static deflection (Figure $6 \mathrm{~h}$ ) is comparable to the static deflection in the absence of the stripes (Figure 6d) might be an argument for the first interpretation. This consideration is based on the following argument: if we assume that a stripe layer is more rigid than a water layer, we expect the static deflection to increase before the tip penetrates the stripes. No significant increase in the static deflection might, therefore, be an indication for the fact that the tip does not penetrate into the stripes. On the other hand, the lateral extension of $4 \mathrm{~nm}$ seen in the data agrees with the stripe width but not with the extension of a water molecule, which would support the second interpretation.

If the first picture is correct, we can compare our slice with the two abovementioned reports from the literature, which have addressed the hydration structure on top of the stripes. In this case, we add yet another observation, as we neither observe none (Yang et al. ${ }^{19}$ ) nor only one (Schlesinger and Sivan ${ }^{17}$ ) vertical layer but at least two vertical layers on top of the stripes. This puzzling observation might indicate the challenging nature of solvation layer mapping. Another possibility to explain the different observations might be that different species are observed in these studies.

\section{CONCLUSIONS}

In this work, we investigate the formation of well-ordered stripes at the graphite-water interface. Despite the large number of reports on these stripes, the origin and fundamental properties of the stripes are discussed controversially in the literature. Here, we demonstrate that stripes are readily formed at the graphite-water interface when plastic syringes are used to place a water droplet on top of the graphite surface. In sharp contrast, when glass syringes are used instead, no stripes form at the interface even though nitrogen was present. This result suggests that the stripes we observed here are composed of contaminations originating from the plastic syringe. Similarly, stripes are readily formed when standard cleaning agents are used during the preparation. To provide detailed structural information for comparison of previous and future stripes observed at the graphite-water interface, we present highresolution AFM data on the domain orientation, stripe widths, internal structure and solvation structure of the stripes. Based on the rich variability of our data, we consider the possibility of several different chemical species being responsible for the stripes. Although we cannot exclude other sources for the stripes presented in the literature, we, however, propose to reconsider earlier assignments to nitrogen in the view of this finding.

\section{ASSOCIATED CONTENT}

\section{Supporting Information}

The Supporting Information is available free of charge at https://pubs.acs.org/doi/10.1021/acs.langmuir.0c00748.

Additional figures with AFM images and 3D AFM data showing stripes at the graphite-water interface (PDF)

\section{AUTHOR INFORMATION}

\section{Corresponding Author}

Angelika Kühnle - Physical Chemistry I, Faculty of Chemistry, Bielefeld University, 33615 Bielefeld, Germany; ○ orcid.org/ 0000-0001-5497-4685; Email: kuehnle@uni-bielefeld.de

\section{Authors}

Sebastian Seibert - Physical Chemistry I, Faculty of Chemistry, Bielefeld University, 33615 Bielefeld, Germany

Stefanie Klassen - Institute of Physical Chemistry, Johannes Gutenberg University Mainz, 55099 Mainz, Germany

Annamaria Latus - Physical Chemistry I, Faculty of Chemistry, Bielefeld University, 33615 Bielefeld, Germany

Ralf Bechstein - Physical Chemistry I, Faculty of Chemistry, Bielefeld University, 33615 Bielefeld, Germany

Complete contact information is available at: https://pubs.acs.org/10.1021/acs.langmuir.0c00748

\section{Notes}

The authors declare no competing financial interest.

\section{ACKNOWLEDGMENTS}

We gratefully acknowledge financial support from the DFG through grant KU1980/7-1.

\section{REFERENCES}

(1) Kozbial, A.; Zhou, F.; Li, Z.; Liu, H.; Li, L. Are Graphitic Surfaces Hydrophobic? Acc. Chem. Res. 2016, 49, 2765-2773.

(2) Dreier, L. B.; Liu, Z.; Narita, A.; van Zadel, M.-J.; Müllen, K.; Tielrooij, K.-J.; Backus, E. H. G.; Bonn, M. Surface-Specific Spectroscopy of Water at a Potentiostatically Controlled Supported Graphene Monolayer. J. Phys. Chem. C 2019, 123, 24031-24038.

(3) Ohno, Y.; Maehashi, K.; Yamashiro, Y.; Matsumoto, K. Electrolyte-Gated Graphene Field-Effect Transistors for Detecting $\mathrm{pH}$ and Protein Adsorption. Nano Lett. 2009, 9, 3318-3322.

(4) Qu, L.; Liu, Y.; Baek, J.-B.; Dai, L. Nitrogen-Doped Graphene as Efficient Metal-Free Electrocatalyst for Oxygen Reduction in Fuel Cells. ACS Nano 2010, 4, 1321-1326.

(5) Lu, Y.-H.; Yang, C.-W.; Hwang, I.-S. Molecular Layer of Gaslike Domains at a Hydrophobic-Water Interface Observed by FrequencyModulation Atomic Force Microscopy. Langmuir 2012, 28, 1269112695.

(6) Lu, Y.-H.; Yang, C.-W.; Hwang, I.-S. Atomic force microscopy study of nitrogen molecule self-assembly at the HOPG-water interface. Appl. Surf. Sci. 2014, 304, 56-64.

(7) Ko, H.-C.; Hsu, W.-H.; Yang, C.-W.; Fang, C.-K.; Lu, Y.-H.; Hwang, I.-S. High-Resolution Characterization of Preferential Gas Adsorption at the Graphene-Water Interface. Langmuir 2016, 32, 11164-11171.

(8) Fang, C.-K.; Ko, H.-C.; Yang, C.-W.; Lu, Y.-H.; Hwang, I.-S. Nucleation processes of nanobubbles at a solid/water interface. Sci. Rep. 2016, 6, 24651. 
(9) Yang, C.-W.; Lu, Y.-H.; Hwang, I. S. Condensation of Dissolved Gas Molecules at a Hydrophobic/Water Interface. Chin. J. Phys. 2013, 51, 174-186.

(10) Wastl, D. S.; Speck, F.; Wutscher, E.; Ostler, M.; Seyller, T.; Giessibl, F. J. Observation of $4 \mathrm{~nm}$ Pitch Stripe Domains Formed by Exposing Graphene to Ambient Air. ACS Nano 2013, 7, 1003210037.

(11) Temiryazev, A.; Frolov, A.; Temiryazeva, M. Atomic-force microscopy study of self-assembled atmospheric contamination on graphene and graphite surfaces. Carbon 2019, 143, 30-37.

(12) Haghighian, N.; Convertino, D.; Miseikis, V.; Bisio, F.; Morgante, A.; Coletti, C.; Canepa, M.; Cavalleri, O. Rippling of graphitic surfaces: a comparison between few-layer graphene and HOPG. Phys. Chem. Chem. Phys. 2018, 20, 13322-13330.

(13) Gallagher, P.; Lee, M.; Amet, F.; Maksymovych, P.; Wang, J.; Wang, S.; Lu, X.; Zhang, G.; Watanabe, K.; Taniguchi, T.; GoldhaberGordon, D. Switchable friction enabled by nanoscale self-assembly on graphene. Nat. Commun. 2016, 7, 10745.

(14) Gallagher, P.; Li, Y.; Watanabe, K.; Taniguchi, T.; Heinz, T. F.; Goldhaber-Gordon, D. Optical Imaging and Spectroscopic Characterization of Self-Assembled Environmental Adsorbates on Graphene. Nano Lett. 2018, 18, 2603-2608.

(15) Beyer, H.; Wagner, T.; Stemmer, A. Length-extension resonator as a force sensor for high-resolution frequency-modulation atomic force microscopy in air. Beilstein J. Nanotechnol. 2016, 7, 432-438.

(16) Teshima, H.; Takata, Y.; Takahashi, K. Adsorbed gas layers limit the mobility of micropancakes. Appl. Phys. Lett. 2019, 115, 071603.

(17) Schlesinger, I.; Sivan, U. Three-Dimensional Characterization of Layers of Condensed Gas Molecules Forming Universally on Hydrophobic Surfaces. J. Am. Chem. Soc. 2018, 140, 10473-10481.

(18) Foster, W.; Aguilar, J. A.; Kusumaatmaja, H.; VoL̈tchovsky, K. In Situ Molecular-Level Observation of Methanol Catalysis at the Water-Graphite Interface. ACS Appl. Mater. Interfaces 2018, 10, 34265-34271.

(19) Yang, C.-W.; Miyazawa, K.; Fukuma, T.; Miyata, K.; Hwang, I.S. Direct comparison between subnanometer hydration structures on hydrophilic and hydrophobic surfaces via three-dimensional scanning force microscopy. Phys. Chem. Chem. Phys. 2018, 20, 23522-23527.

(20) Schlesinger, I.; Sivan, U. New Information on the Hydrophobic Interaction Revealed by Frequency Modulation AFM. Langmuir 2017, 33, 2485-2496.

(21) Ball, P. Water as an active constituent in cell biology. Chem. Rev. 2008, 108, 74-108.

(22) Rode, S.; Stark, R.; Lübbe, J.; Tröger, L.; Schütte, J.; Umeda, K.; Kobayashi, K.; Yamada, H.; Kühnle, A. Modification of a commercial atomic force microscopy for low-noise, high-resolution frequency-modulation imaging in liquid environment. Rev. Sci. Instrum. 2011, 82, 073703.

(23) Söngen, H.; Nalbach, M.; Adam, H.; Kühnle, A. Threedimensional atomic force microscopy mapping at the solid-liquid interface with fast and flexible data acquisition. Rev. Sci. Instrum. 2016, 87, 063704.

(24) Adam, H.; Rode, S.; Schreiber, M.; Kobayashi, K.; Yamada, H.; Kühnle, A. Photothermal excitation setup for a modified commercial atomic force microscope. Rev. Sci. Instrum. 2014, 85, 023703.

(25) Berkelaar, R. P.; Dietrich, E.; Kip, G. A. M.; Kooij, E. S.; Zandvliet, H. J. W.; Lohse, D. Exposing nanobubble-like objects to a degassed environment. Soft Matter 2014, 10, 4947-4955.

(26) Flores, M.; Cisternas, E.; Correa, J. D.; Vargas, P. Moiré patterns on STM images of graphite induced by rotations of surface and subsurface layers. Chem. Phys. 2013, 423, 49-54.

(27) Chang, H.; Bard, A. J. Observation and characterization by scanning tunneling microscopy of structures generated by cleaving highly oriented pyrolytic graphite. Langmuir 1991, 7, 1143-1153.

(28) Luican-Mayer, A.; Barrios-Vargas, J. E; Falkenberg, J. T.; Autes, G.; Cummings, A. W; Soriano, D.; Li, G.; Brandbyge, M.; Yazyev, O. V; Roche, S.; Andrei, E. Y Localized electronic states at grain boundaries on the surface of graphene and graphite. 2D Mater. 2016, 3, 031005.

(29) Voïtchovsky, K.; Giofrè, D.; Segura, J. J.; Stellacci, F.; Ceriotti, $M$. Thermally-nucleated self-assembly of water and alcohol into stable structures at hydrophobic interfaces. Nat. Commun. 2016, 7, 13064.

(30) Suzuki, K.; Oyabu, N.; Kobayashi, K.; Matsushige, K.; Yamada, $\mathrm{H}$. Atomic-Resolution Imaging of Graphite-Water Interface by Frequency Modulation Atomic Force Microscopy. Appl. Phys. Express 2011, 4, 125102.

(31) Söngen, H.; Jaques, Y. M.; Zivanovic, L.; Seibert, S.; Bechstein, R.; Spijker, P.; Onishi, H.; Foster, A. S.; Kühnle, A. Hydration layers at the graphite-water interface: Attraction or confinement. Phys. Rev. B 2019, 100, 205410.

(32) Marutschke, C.; Walters, D.; Cleveland, J.; Hermes, I.; Bechstein, R.; Kühnle, A. Three-dimensional hydration layer mapping on the (10.4) surface of calcite using amplitude modulation atomic force microscopy. Nanotechnology 2014, 25, 335703. 